\title{
HERWIRI1.031: New Approach to Parton Shower MC's in Precision QCD Theory
}

\author{
B.F.L. Ward ${ }^{* \dagger}$ \\ Baylor University \\ E-mail: BFL_Ward@baylor.edu
}

\author{
S.A. Yost \\ The Citadel \\ E-mail: scott.yostecitadel.edu
}

The new IR-improved Dokshitzer-Gribov-Lipatov-Altarelli-Parisi-Callan-Symanzik (DGLAPCS) kernels recently developed by one of us is implemented in the HERWIG6.5 environment to generate a new MC, HERWIRI1.0(31), for hadron-hadron scattering at high energies. The comparison between the parton shower generated by the standard DGLAP-CS kernels and that generated by the new IR-improved DGLAP-CS kernels is illustrated using MC data. This is done for some of the respective exact $\mathscr{O}\left(\alpha_{s}\right)$ corrected spectra using the seamless interfaces to MC@NLO while making comparisons with FNAL data. Some discussion of possible implications for LHC phenomenology is also presented.

35th International Conference of High Energy Physics - ICHEP2010,

July 22-28, 2010

Paris, France

\footnotetext{
${ }^{*}$ Speaker.

$\dagger$ Work supported in part by US DOE grant DE-FG02-09ER41600.

†Work supported in part by US DOE grant DE-SC0000528 and The Citadel Foundation.
} 
Resummed $\mathscr{O}\left(\alpha_{s}^{2} L^{n}\right), \mathscr{O}\left(\alpha_{s} \alpha L^{n^{\prime}}\right), \mathscr{O}\left(\alpha^{2} L^{n^{\prime \prime}}\right)$ corrections for $n=0,1,2, n^{\prime}=0,1,2, n^{\prime \prime}=1,2$, in the presence of parton showers, on an event-by-event basis, without double counting and with exact phase space are required $[1,2]$ for precision LHC physics $(1 \%$ or better total theoretical precision [3]). We present here the first step in realizing our new MC event generator approach to such physics with amplitude-based $\mathrm{QED} \otimes \mathrm{QCD}$ resummation [4] in the HERWIG6.5 [5] environment by our new parton shower MC for QCD, HERWIRI1.0(31) [6]. HERWIRI1.0(31) already shows improvement in comparison with the FNAL rapidity and soft $p_{T}$ data on single $Z$ production as we quantify below. While the explicit IR cut-offs in the HERWIG6.5 environment will not be removed here, HERWIRI only involves integrable distributions so that these cut-offs could be removed.

We first review our approach to resummation, which can be shown $[6,7]$ to be equivalent to those in Refs. $[8,9]$, before we turn to a summary of the attendant new IR-improved DGLAPCS $[10,11]$ theory [7] and a description of the implementation of the new IR-improved kernels in the framework of HERWIG6.5 [5] to arrive at HERWIRI1.0(31). We illustrate the effects of the IR-improvement and compare with recent data from FNAL ${ }^{1}$.

In Refs. $[4,7]$ we have derived the following expression for the hard cross sections in the SM $S U_{2 L} \times U_{1} \times S U_{3}^{c}$ EW-QCD theory

$$
\begin{gathered}
d \hat{\sigma}_{\exp }=e^{\mathrm{SUM}_{\mathrm{IR}}(\mathrm{QCED})} \sum_{n, m=0}^{\infty} \frac{1}{n ! m !} \int \frac{d^{3} p_{2}}{p_{2}^{0}} \frac{d^{3} q_{2}}{q_{2}^{0}} \prod_{j_{1}=1}^{n} \frac{d^{3} k_{j_{1}}}{k_{j_{1}}} \prod_{j_{2}=1}^{m} \frac{d^{3} k_{j_{2}}^{\prime}}{k_{j_{2}}^{\prime}} \\
\times \int \frac{d^{4} y}{(2 \pi)^{4}} e^{i y \cdot\left(p_{1}+q_{1}-p_{2}-q_{2}-\sum k_{j_{1}}-\sum k_{j_{2}}^{\prime}\right)+D_{\mathrm{QCED}}} \tilde{\bar{\beta}}_{n, m}\left(k_{1}, \ldots, k_{n} ; k_{1}^{\prime}, \ldots, k_{m}^{\prime}\right),
\end{gathered}
$$

where the new YFS-style [13] residuals $\tilde{\bar{\beta}}_{n, m}\left(k_{1}, \ldots, k_{n} ; k_{1}^{\prime}, \ldots, k_{m}^{\prime}\right)$ have $n$ hard gluons and $m$ hard photons and we illustrate the generic $2 \mathrm{f}$ final state with momenta $p_{2}, q_{2}$ specified for definiteness. The infrared functions $\mathrm{SUM}_{\mathrm{IR}}(\mathrm{QCED}), D_{\mathrm{QCED}}$ are defined in Refs. [4, 7]. Eq. (1) is exact to all orders in $\alpha$ and in $\alpha_{s}$.

The result Eq. (1) allows us to improve [7] in the IR regime the DGLAP-CS [10, 11] kernels as follows, using a standard notation:

$$
\begin{aligned}
P_{q q}^{e x p}(z)= & C_{F} F_{\mathrm{Y} F S}\left(\gamma_{q}\right) e^{\frac{1}{2} \delta_{q}}\left[\frac{1+z^{2}}{1-z}(1-z)^{\gamma_{q}}-f_{q}\left(\gamma_{q}\right) \delta(1-z)\right], \\
P_{G q}^{e x p}(z)= & C_{F} F_{\mathrm{Y} F S}\left(\gamma_{q}\right) e^{\frac{1}{2} \delta_{q}} \frac{1+(1-z)^{2}}{z} z^{\gamma_{q}}, \\
P_{G G}^{e x p}(z)= & 2 C_{G} F_{\mathrm{Y} F S}\left(\gamma_{G}\right) e^{\frac{1}{2} \delta_{G}}\left\{\frac{1-z}{z} z^{\gamma_{G}}+\frac{z}{1-z}(1-z)^{\gamma_{G}}\right. \\
& \left.\quad+\frac{1}{2}\left(z^{1+\gamma_{G}}(1-z)+z(1-z)^{1+\gamma_{G}}\right)-f_{G}\left(\gamma_{G}\right) \delta(1-z)\right\}, \\
P_{q G}^{e x p}(z)= & F_{\mathrm{Y} F S}\left(\gamma_{G}\right) e^{\frac{1}{2} \delta_{G}} \frac{1}{2}\left\{z^{2}(1-z)^{\gamma_{G}}+(1-z)^{2} z^{\gamma_{G}}\right\},
\end{aligned}
$$

where the superscript "exp" indicates that the kernel has been resummed as predicted by Eq. (1) when it is restricted to QCD alone and where we refer the reader to Refs. [7] for the detailed

\footnotetext{
${ }^{1}$ From Ref. [12] the current state-of-the-art theoretical precision tag on single $Z$ production at the LHC at $14 \mathrm{TeV}$ is $(4.91 \pm 0.38) \%=(2.45 \pm 0.73) \%(Q C D+E W) \oplus 4.11 \%(P D F) \oplus 1.10 \pm 0.44 \%(Q C D S c a l e)$ and for single $\mathrm{W}^{+}\left(\mathrm{W}^{-}\right)$ $5.05 \pm 0.58 \%(5.24 \pm 0 \%)$.
} 
definitions of the respective resummation functions $F_{\mathrm{Y} F S}, \gamma_{A}, \delta_{A}, f_{G}, A=q, G^{2}$. See Refs. [4, 7] for discussion of illustrative results and implications of the new kernels and Eq. (1) that are beyond the scope we have here.

We have implemented the new IR-improved kernels in the HERWIG6.5 environment to produce a new MC, HERWIRI1.0, which stands for "high energy radiation with IR improvement"3. We modify the kernels in the HERWIG6.5 module HWBRAN and in the attendant related modules ${ }^{4}$ with the following substitutions: DGLAP-CS $P_{A B} \Rightarrow$ IR-I DGLAP-CS $P_{A B}^{\exp }$ while leaving the hard processes alone for the moment. We have in progress [16]the inclusion in our framework of YFS synthesized electroweak modules from Refs. [17]for HERWIG6.5, HERWIG++ [18] and MC@ NLO [19] hard processes ${ }^{5}$, as the CTEQ [22] and MRST(MSTW) [23] best (after 2007) parton densities do not include the precision electroweak higher order corrections that are needed in a $1 \%$ precison tag budget for processes such as single heavy gauge boson production in the LHC environment [2].

The details of the implementation are given in Refs. $[4,6]$ and we do not reproduce them here due to a lack of space. We have done many comparisons of the properties of the parton showers from HERWIG6.510 and HERWIRI1.031. In general, the IR-improved showers tend to be softer in the energy fraction variable $z=E / E_{\text {Beam }}$ where $E\left(E_{\text {Beam }}\right)$ is the cms parton(beam) energy for hadron-hadron scattering respectively. See Refs. [4, 6] for the complete discussion of such comparisons. We show in Fig. 1 [6] comparison analyses with the data from FNAL on the $Z$ rapidity and $p_{T}$ spectra as reported in Refs. [24, 25]. We see that HERWIRI1.0(31) and HERWIG6.5 both give a reasonable overall representation of the CDF rapidity data but that HERWIRI1.031 is somewhat closer to the data for small values of $Y$ (The $\chi^{2} /$ d.o.f is $1.77(1.54)$ for HERWIG6.5 (HERWIRI1.0(31)).). Including the NLO contributions to the hard process via MC@NLO/HERWIG6.510 and MC@NLO/HERWIRI1.031[19 $]^{6}$ improves the agreement for both HERWIG6.510 and for HERWIRI1.031 (the $\chi^{2} /$ d.o.f are changed to 1.40 and 1.42 respectively). That they are both consistent with one another and within $10 \%$ of the data in the low $Y$ region is fully consistent with expectations and is an important cross-check on our work. A more precise discussion at the NNLO level with DGLAP-CS IR-improvement and a more complete discussion of the errors will appear [26]. We also see that HERWIRI1.031 gives a better fit to the D0 $p_{T}$ data compared to HERWIG6.510 for low $p_{T}$, (for $p_{T}<12.5 \mathrm{GeV}$, the $\chi^{2} /$ d.o.f. are $\sim 2.5$ and 3.3 respectively if we add the statistical and systematic errors), showing that the IR-improvement makes a better representation of QCD in the soft regime for a given fixed order in perturbation theory. Adding the $\mathscr{O}\left(\alpha_{s}\right)$ correction from MC@NLO [19] improves the $\chi^{2} /$ d.o.f for the HERWIRI1.031 in both the soft and hard regimes and it improves the HERWIG6.510 $\chi^{2} /$ d.o.f for $p_{T}$ near $3.75 \mathrm{GeV}$ where the distribution peaks. For $p_{T}<7.5 \mathrm{GeV}$ the $\chi^{2} /$ d.o.f for the MC@NLO/HERWIRI1.031 is 1.5 whereas that for MC@NLO/HERWIG6.510 is worse. We await further tests of the new approach, both at FNAL and at LHC. - One of us (B.F.L.W) acknowledges helpful discussions with Prof. Bryan Webber and Prof. M. Seymour and with Prof. S. Frixione. B.F.L. Ward also thanks Prof. L. Alvarez-Gaume and Prof. W. Hollik for the support

\footnotetext{
${ }^{2}$ The improvement in Eq. (2) should be distinguished from the resummation in parton density evolution for the “ $z \rightarrow 0$ " Regge regime - see for example Ref. $[14,15]$. This latter improvement must also be taken into account for precision LHC predictions.

${ }^{3}$ We thank M. Seymour and B. Webber for helpful discussion on this point.

${ }^{4}$ We thank M. Seymour and B. Webber for helpful discussion.

${ }^{5}$ Similar results for PYTHIA [20] and for the new kernel evolution in Ref. [21] are under study.

${ }^{6}$ We thank S. Frixione for helpful discussions with this implementation.
} 
(a)

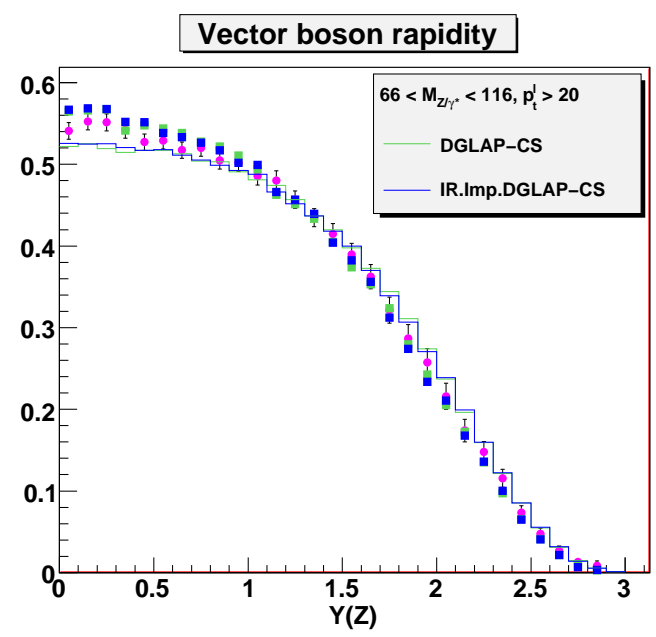

(b)



Figure 1: Comparison with FNAL data: (a), CDF rapidity data on $\left(Z / \gamma^{*}\right)$ production to $e^{+} e^{-}$pairs, the circular dots are the data, the green(blue) lines are HERWIG6.510(HERWIRI1.031); (b), D0 $p_{T}$ spectrum data on $\left(Z / \gamma^{*}\right)$ production to $e^{+} e^{-}$pairs, the circular dots are the data, the blue triangles are HERWIRI1.031, the green triangles are HERWIG6.510 - in both (a) and (b) the blue squares are MC@NLO/HERWIRI1.031, and the green squares are MC@NLO/HERWIG6.510. These are untuned theoretical results.

and kind hospitality of the CERN TH Division and of the Werner-Heisenberg Institut, MPI, Munich, respectively, while this work was in progress. S. Yost acknowledges the hospitality and support of Princeton University and the CERN TH Division.

\section{References}

[1] S. Haywood, P.R. Hobson, W. Hollik and Z. Kunszt, in Proc. 1999 CERN Workshop on Standard Model Physics ( and more ) at the LHC, CERN-2000-004, eds. G. Altarelli and M.L. Mangano,( CERN, Geneva, 2000 ) p. 122; H. Spiesberger, Phys. Rev. D52 ( 1995 ) 4936; W.J.

Stirling,"Electroweak Effects in Parton Distribution Functions", talk presented at ESF Exploratory Workshop, Electroweak Radiative Corrections to Hadronic Observables at TeV Energies, Durham, Sept., 2003; M. Roth and S. Weinzierl,Phys. Lett. B590 (2004) 190; J. Blumlein and H. Kawamura, Nucl. Phys. $B 708$ (2005) 467; Acta Phys. Pol. B33 (2002) 3719; W. J. Stirling et al., in Proc.

ICHEP04, eds. H. Chen et al. (World Sci. Publ., Singapore, 2005) p. 527; A.D. Martin et al., Eur. Phys. J. C39 (2005) 155, and references therein.

[2] A. Kulesza et al., PoS RADCOR2007: 001, 2007; A. Denner et al., PoS RADCOR2007: 002, 2007; Nucl.Phys. B662 (2003) 299; G. Balossini et al., arXiv:0805.1129; S. Dittmaier, in Proc. LP09, 2009, in press, and references therein.

[3] See for example S. Jadach et al., in Geneva 1995, Physics at LEP2, vol. 2, pp. 229-298; hep-ph/9602393, for a discussion of technical and physical precision.

[4] C. Glosser, S. Jadach, B.F.L. Ward and S.A. Yost,Mod. Phys. Lett. A19(2004) 2113; B.F.L. Ward, C. Glosser, S. Jadach and S.A. Yost, in Proc. ICHEP04, vol. 1, eds. H. Chen et al.,(World. Sci. Publ. Co., 
Singapore, 2005) p. 588; B.F.L. Ward and S. Yost, Acta Phys. Polon. B38 (2007) 2395; PoS RADCOR2007: 038, 2007; B.F.L. Ward et al., in Proc. ICHEP08, Philadelphia, 2008, eConf C080730, [arXiv:0810.0723]; in Proc. 2008 HERA-LHC Workshop,DESY-PROC-2009-02, eds. H. Jung and A. De Roeck, (DESY, Hamburg, 2009)pp. 180-186, and references therein.

[5] G. Corcella et al., preprint hep-ph/0210213; J. High Energy Phys. 0101 (2001) 010; G. Marchesini et al., Comput. Phys. Commun.67 (1992) 465.

[6] S. Joseph et al., Phys. Lett. B685, 283 (2010); Phys. Rev. D81, 076008 (2010).

[7] B.F.L. Ward, Adv. High Energy Phys. 2008 (2008) 682312; Ann. Phys. 323 (2008) 2147.

[8] G. Sterman,Nucl. Phys. B281, 310 (1987); S. Catani and L. Trentadue, Nucl. Phys. B327, 323 (1989); ibid. B353, 183 (1991).

[9] See for example C. W. Bauer, A.V. Manohar and M.B. Wise, Phys. Rev. Lett. 91 (2003) 122001; Phys. Rev. D70 (2004) 034014.

[10] G. Altarelli and G. Parisi, Nucl. Phys. B126 (1977) 298; Yu. L. Dokshitzer, Sov. Phys. JETP 46 (1977) 641; L. N. Lipatov, Yad. Fiz. 20 (1974) 181; V. Gribov and L. Lipatov, Sov. J. Nucl. Phys. 15 (1972) 675; V. Gribov and L. Lipatov, Sov. J. Nucl. Phys. 15 (1972) 938; see also J.C. Collins and J. Qiu, Phys. Rev. D39 (1989) 1398 for an alternative discussion of DGLAP-CS theory.

[11] C.G. Callan, Jr., Phys. Rev. D2 (1970) 1541; K. Symanzik, Commun. Math. Phys. 18 (1970) 227; K. Symanzik, in Springer Tracts in Modern Physics, 57, ed. G. Hoehler (Springer, Berlin, 1971) p. 222; see also S. Weinberg, Phys. Rev. D8 (1973) 3497.

[12] N.E. Adam et al., J. High Energy Phys. 05 (2008) 062; ibid. 09 (2008) 133; ibid. 11 (2010) 074.

[13] D. R. Yennie, S. C. Frautschi, and H. Suura, Ann. Phys. 13 (1961) 379; see also K. T. Mahanthappa, Phys. Rev. 126 (1962) 329.

[14] B.I. Ermolaev, M. Greco and S.I. Troyan, PoS DIFF2006 (2006) 036, and references therein.

[15] G. Altarelli, R.D. Ball and S. Forte, PoS RADCOR2007 (2007) 028.

[16] M. Hejna et al., to appear.

[17] S. Jadach and B.F.L. Ward, Comput. Phys. Commun. 56(1990) 351; Phys.Lett. B274 (1992) 470; S. Jadach et al., Comput. Phys. Commun. 102 (1997) 229; S. Jadach, B.F.L. Ward and Z. Was, Phys. Rev. D 63 (2001) 113009; Comp. Phys. Commun. 130 (2000) 260; S. Jadach et al., ibid.140 (2001) 432, 475 , and references therein.

[18] M. Bahr et al., arXiv:0812.0529 and references therein.

[19] S. Frixione and B.Webber, J. High Energy Phys. 0206 (2002) 029; S. Frixione, P. Nason and B. Webber, ibid. 0308 (2003) 007.

[20] T. Sjostrand et al., hep-ph/0308153.

[21] S. Jadach and M. Skrzypek, Comput. Phys. Commun. 175 (2006) 511; P. Stevens et al., Acta Phys. Polon. B38 (2007) 2379, and references therein.

[22] F. Olness, private communication; P.M. Nadolsky et al., arXiv:0802.0007.

[23] R. Thorne, private communication; A.D. Martin et al., arXiv:0901.0002 and references therein.

[24] C. Galea, in Proc. DIS 2008, London, 2008, http://dx.doi.org/10.3360/dis.2008.55.

[25] V.M. Abasov et al., Phys. Rev. Lett. 100, 102002 (2008).

[26] B.F.L. Ward et al., to appear. 